\title{
Optimal conditions for the storage of German cockroach extract
}

\author{
KYOUNG YONG JEONG ${ }^{1}$, JONGSUN LEE ${ }^{1}$, JI-EUN YUK ${ }^{1}$, KYUNG HEE PARK ${ }^{1}$, \\ JAE-HYUN LEE ${ }^{1}$, JUNG DONG KIM ${ }^{2}$ and JUNG-WON PARK ${ }^{1,2}$ \\ ${ }^{1}$ Department of Internal Medicine, Institute of Allergy, Yonsei University College of Medicine, Seoul 03722; \\ ${ }^{2}$ Prolagen, Seoul 03722, Republic of Korea
}

Received June 27, 2018; Accepted November 1, 2019

DOI: $10.3892 / \mathrm{mmr} .2019 .10854$

\begin{abstract}
Allergen extracts are commonly utilized for diagnosis and immunotherapy; however, the stability of protease-rich extracts is important for a precise diagnosis and treatment efficacy. The present study determines the optimal conditions for the storage of German cockroach allergen extract. Cockroach extracts were reconstituted in four buffers: normal saline (NS), $50 \%$ glycerol in NS, $0.3 \%$ phenol in NS, or $0.3 \%$ phenol and $50 \%$ glycerol in NS. The extracts in different buffers were stored either at room temperature $\left(18-26^{\circ} \mathrm{C}, \mathrm{RT}\right)$ or refrigerated $\left(2-8^{\circ} \mathrm{C}\right)$. Subsequently, the protein concentration and allergen content (Bla g 1 and Bla g 2) in the extracts were examined for the course of one year. Extract potency was estimated by inhibition ELISA. At least $90.5 \%$ protein, $94.4 \%$ Bla g 1, 65.2\% Bla g 2, and $91.4 \%$ potency remained after one year when $50 \%$ glycerol NS was added to the extract with refrigeration. However, less than $13.7 \%$ protein, $17.1 \%$ Bla g 1, $0 \%$ Bla g 2 and $32.5 \%$ potency were maintained after one year when 50\% glycerol NS was not added to the extract and was maintained at RT. The addition of $0.3 \%$ phenol NS did not show significant effects on extract stability. The addition of $50 \%$ glycerol NS and refrigerated storage temperature were found to be important factors for increasing the shelf life of protease-rich cockroach extract.
\end{abstract}

\section{Introduction}

Cockroach sensitization is a risk factor for asthma. Protease-activated receptors (PAR)-2, Toll-like receptors (TLRs) and C-type lectin receptors have been suggested to play a role in the penetration of cockroach allergens through epithelial cells and allergic inflammation (1). Strong protease activity in cockroach extracts can degrade protein components within the extract. Dilution and mixture of cockroach

Correspondence to: Professor Jung-Won Park, Department of Internal Medicine, Institute of Allergy, Yonsei University College of Medicine, 50-1 Yonsei-ro, Seodamun-gu, Seoul 03722, Republic of Korea

E-mail: parkjw@yuhs.ac

Key words: allergen extract, cockroach, stability extract with pollen extracts was reported to cause a loss of allergen potency (2). The additions of protease-inhibitor $(\varepsilon$-aminocaproic acid) and sucrose were reported to increase the shelf life of the protease-rich American cockroach extract (3); however, the potential side effects of this protease inhibitor have not been examined.

More recently, mixtures of cockroach allergens with other indoor allergens for immunotherapy have been found useful when more than $50 \%$ glycerol is included, even though current immunotherapy guidelines recommend the separation of high protease extracts from other products if possible $(4,5)$. Moreover, cockroach extracts for immunotherapy are currently not standardized since allergen and protein contents in commercial extracts vary greatly, and the potency of commercial extracts was estimated as 10 to 8,570 bioequivalent units (BAU) $/ \mathrm{ml}(6)$.

In the present study, we determined the optimal storage conditions for protease-rich German cockroach extracts prepared in a standardized manner using a Korean isolate (7). The changes in protein and major allergen (Bla g 1 and Bla $\mathrm{g} 2$, respectively) contents as well as allergen potency (total IgE reactivity) in the extract stored under various conditions were examined over a 1-year time period.

\section{Materials and methods}

Serum samples. Serum samples were collected from 15 patients (age range 14-54 years, mean 32 years, 9 males and 6 females) at the Allergy-Asthma Center at Severance Hospital (Seoul, Korea) between November 2014 and December 2018. Patient consent was obtained before the blood collection. Specific IgE to German cockroach was determined using the ImmunoCAP system (Thermo Fisher Scientific, Inc.) and serum samples with specific IgE levels higher than $3.5 \mathrm{kU}_{\mathrm{A}} / 1$ were selected. A pooled serum sample from 13 healthy subjects (age range 25-81 years, mean 48 years, 9 males and 10 females) was used as a negative control. samples were taken between March 19, 2014 and May 6, 2016 at Severance Hospital, Seoul, Korea. The Institutional Review Board of the Yonsei University Health System approved this study (no. 4-2013-0397).

Allergen extraction. Allergen extract was prepared as previously described (7). In brief, lyophilized cockroach was homogenized in liquid nitrogen, defatted with ethyl ether, 
extracted in phosphate-buffered saline (PBS), dialyzed against distilled water (DW), filtered $(0.22 \mu \mathrm{m})$ and lyophilized again. The protein concentration was determined by Bradford assay (Bio-Rad Laboratories) after dissolution in appropriate buffers.

The lyophilized extract was reconstituted in one of four solutions: normal saline (NS), $50 \%$ glycerol in NS, $0.3 \%$ phenol in NS, or $0.3 \%$ phenol and $50 \%$ glycerol in NS. The dissolved extracts were aliquoted and maintained at room temperature (RT, $\left.18-26^{\circ} \mathrm{C}\right)$ or refrigerated $\left(2-8^{\circ} \mathrm{C}\right)$. Samples were taken for testing at weeks $1,2,4,9,13,26$ and 52 .

Protein analyses and measurement of allergen content. Protein concentration was measured via Bradford assay using bovine serum albumin (BSA) as the standard. Allergen content (Bla g 1 and Bla g 2) was assessed using two-site ELISA (Indoor Biotechnologies Inc.).

IgE antibody binding inhibition assay. Allergen extract potency was compared using inhibition ELISA. Allergen extract $(100 \mu \mathrm{l})$ at week 0 was coated overnight at $4^{\circ} \mathrm{C}$ at a $10 \mu \mathrm{g} / \mathrm{ml}$ concentration in $50 \mathrm{mM}$ carbonate buffer, $\mathrm{pH} 9.6$. Simultaneously, 4-fold diluted pooled serum from German cockroach allergy patients $(n=15)$ was pre-incubated overnight at $4^{\circ} \mathrm{C}$ with the extract of interest (1/10 volume) or $1 \%$ BSA solution as a control. IgE antibodies were detected with biotinylated goat anti-human $\operatorname{IgE}(\varepsilon$ chain specific; Vector Laboratories Inc.) and streptavidin-peroxidase (Sigma-Aldrich). The inhibition percentage was calculated as $\left(1-A_{i} / A_{0}\right) \times 100$, where $A_{i}$ indicates the absorbance at $450 \mathrm{~nm}$ with an inhibitor and $\mathrm{A}_{0}$ indicates the absorbance without an inhibitor.

SDS-PAGE and IgE immunoblotting. Protein profile and IgE reactive components were examined by SDS-PAGE and $\operatorname{IgE}$ immunoblotting. Proteins $(20 \mu \mathrm{l})$ were separated on $12 \%$ SDS-PAGE gels under reducing conditions. Gels were stained with Coomassie brilliant blue or transferred onto a polyvinylidene difluoride (PVDF) membrane (GE Waters \& Process Technologies). IgE reactive components were probed with alkaline phosphate conjugated goat anti-human $\operatorname{IgE}$ (1:1,000-dilution) ( $\varepsilon$ chain specific; Sigma-Aldrich), and color development was conducted with nitro blue tetrazolium and 3-bromo-4-chloro-5-indolyl-phosphate (Promega).

Statistical analyses. Statistical differences between test groups were analyzed by two-way ANOVA followed by Bonferroni correction. $\mathrm{P}<0.05$ was considered to indicate a statistically significant difference.

\section{Results}

Protein content in German cockroach extract. Initial protein concentration was determined to be $0.4 \mathrm{mg} / \mathrm{ml}$. Approximately $33.8 \%$ of the original protein concentration was detected after 52 weeks when $50 \%$ glycerol was added to the extract in NS, whereas $4.1 \%$ remained in the extracts of NS at RT (Fig. 1A). When refrigerated, $>90.0 \%$ of the protein was detected when $50 \%$ glycerol NS was added, while $<78.0 \%$ was detected without glycerol (Fig. 1B).
Allergen content in German cockroach extract. Initial concentrations of Bla g 1 and Bla g 2 were 35.3 and $7.0 \mu \mathrm{g} / \mathrm{ml}$, respectively. Blag 1 content in the extract stored at RT remained at $17.1 \%$ in NS, $7.0 \%$ in $0.3 \%$ phenol NS, $100 \%$ in $50 \%$ glycerol NS, and 97.1\% in glycerol phenol NS (Fig. 2A). Bla g 1 content detected in the refrigerated extracts was $96.4 \%$ in NS, $100 \%$ in $50 \%$ glycerol NS, $88.0 \%$ in $0.3 \%$ phenol NS and $94.4 \%$ in glycerol with phenol NS (Fig. 2B). A similar pattern of Bla g 2 content was observed, even though Bla g 1 in the extract was shown to be more stable than Bla g 2 (Fig. 2C and D).

Allergen potency of German cockroach extract. Potency was retained at $73.0 \%$ in $50 \%$ glycerol NS and $66.9 \%$ in $50 \%$ glycerol with phenol NS when stored at RT, whereas $26.9 \%$ in NS and 32.5\% in phenol NS were retained in the absence of glycerol (Fig. 3A). When refrigerated, $91.4 \%$ potency in 50\% glycerol NS and $92.6 \%$ potency in 50\% glycerol with phenol NS of potency was maintained, while $72.9 \%$ of the allergen potency in NS and $74.9 \%$ in phenol NS were retained in the absence of glycerol (Fig. 3B).

Change of protein profile and IgE reactive components. Notably, a 35-kDa component and components $>55 \mathrm{kDa}$ were shown to be degraded when the extracts were maintained at RT (Fig. 4A). Slow disruption of the $35 \mathrm{kDa}$ band was observed in the refrigerated extract in 50\% glycerol NS and also bands around $70 \mathrm{kDa}$ in the refrigerated extract in $0.3 \%$ phenol NS (Fig. 4B). Notably, some partially degraded proteins produced bands of $\sim 55 \mathrm{kDa}$ in $50 \%$ glycerol NS at RT, $50 \%$ glycerol with $0.3 \%$ phenol NS at RT, and $50 \%$ glycerol with $0.3 \%$ phenol NS and refrigerated, $\sim 38 \mathrm{kDa}$ in $0.3 \%$ phenol NS at RT.

Strong $\mathrm{IgE}$ reactions were detected between 55 and $100 \mathrm{kDa}$ components when extracts were dissolved in a solution with $50 \%$ glycerol NS (regardless of $0.3 \%$ phenol) and kept refrigerated (Fig. 4B, C and D). IgE reaction to these components seemed to correlate with potency. IgE reactivity to the $34 \mathrm{kDa}$ band was detected only in the refrigerated samples and within the first few weeks of the sampling at RT (Fig. 4C and D).

No IgE reactive component was detected by IgE immunoblotting with the extracts at week 0 , which should include all the allergenic components (Fig. 4E and G), using a pooled serum sample with healthy subjects (Fig. 4F and $\mathrm{H}$ ).

\section{Discussion}

Commercial cockroach extracts are highly variable in terms of allergen content and potency. However, high protease activity in cockroach extracts makes it more difficult to standardize the allergen extracts. Glycerol is a well-known stabilizer of allergen extracts (8). However, stability of German cockroach extract has not been described. Therefore, we aimed to observe what occurs with German cockroach extract which has strong protease activity. In this study, the protein concentration was well conserved when refrigerated, and the addition of $50 \%$ glycerol NS seemed to inhibit the protease activity and enhanced cockroach extract stability.

The change of $\mathrm{IgE}$ reactive components were investigated by SDS-PAGE and IgE immunoblotting. However, it was not certain concerning the identity of each protein band. It seems that a high salt concentration affects the solubility of various 
A

German cockroach extract at room temperature

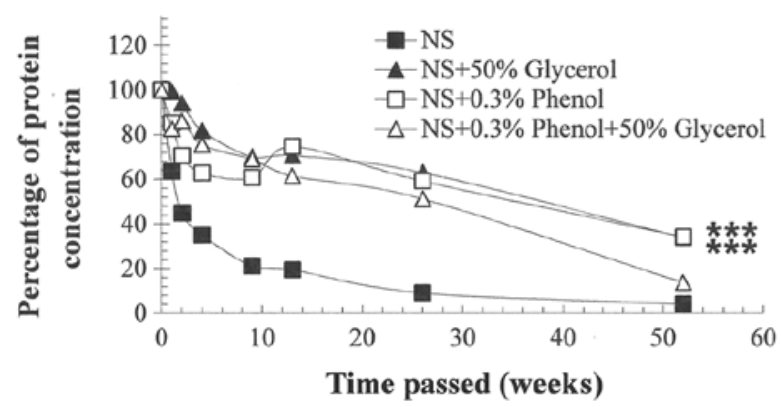

B German cockroach extract at $4^{\circ} \mathrm{C}$

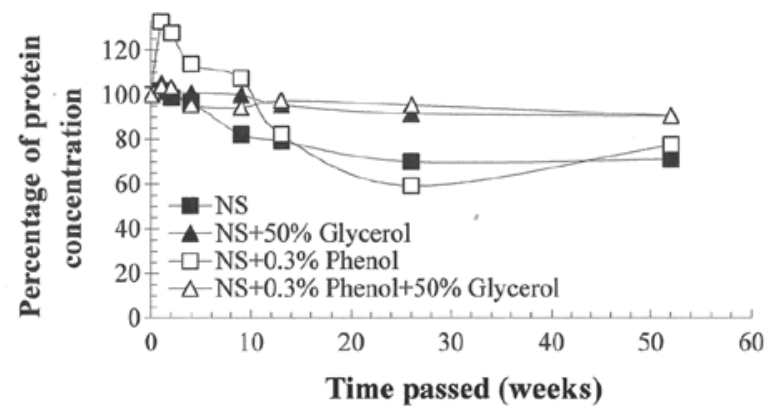

Figure 1. Change of protein concentration in cockroach extract over one year of storage under various conditions. Protein concentration was determined using the Bradford assay in the extracts kept at (A) room temperature and (B) refrigerated. ${ }^{* * *} \mathrm{P}<0.001$. NS, normal saline.

A

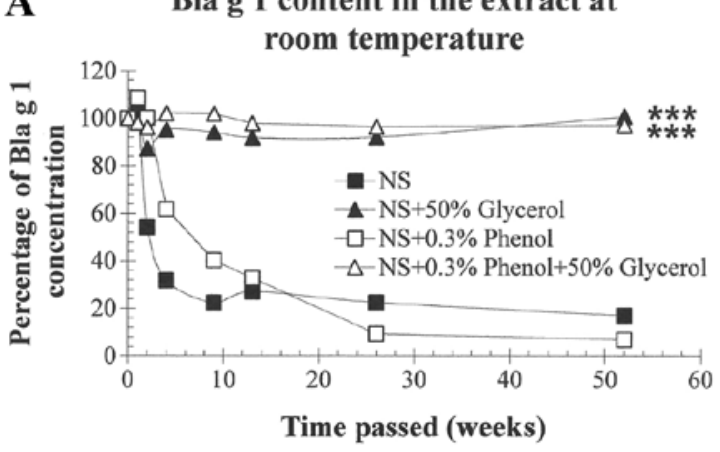

C

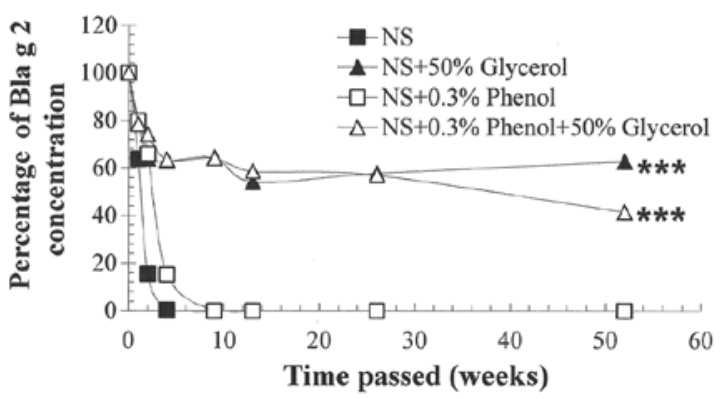

B Bla g 1 content in the extract at $4^{\circ} \mathrm{C}$

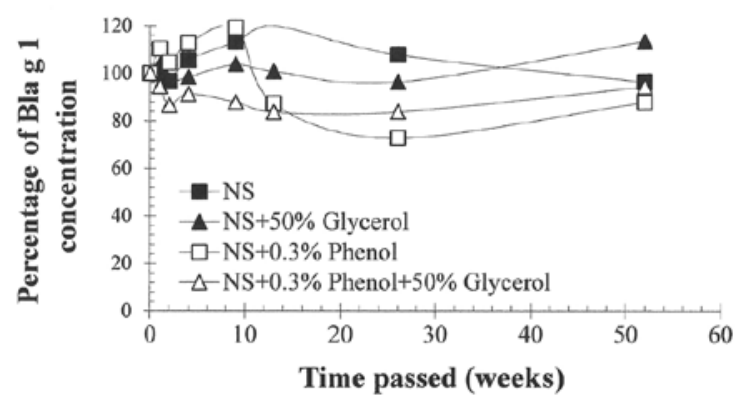

D Bla 2 content in the extract at $4^{\circ} \mathrm{C}$

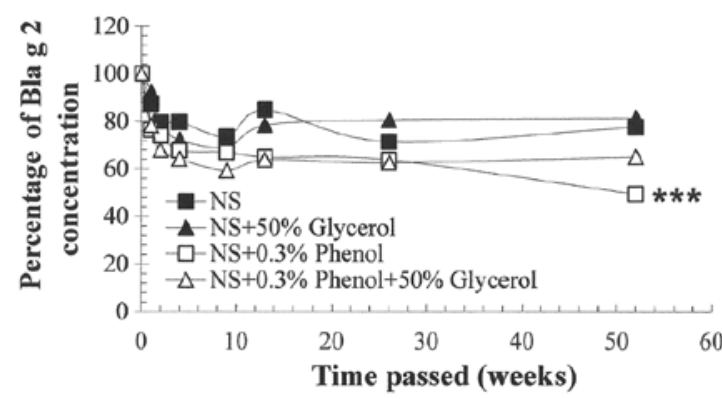

Figure 2. Change of Bla g 1 and Bla g 2 content in cockroach extract over one year of storage at various conditions. Bla g 1 content in the extract stored at (A) room temperature and (B) refrigerated, Bla g 2 content at (C) room temperature, and (D) refrigerated, measured by a two-site ELISA. ${ }^{* * * *} \mathrm{P}<0.001$. NS, normal saline.

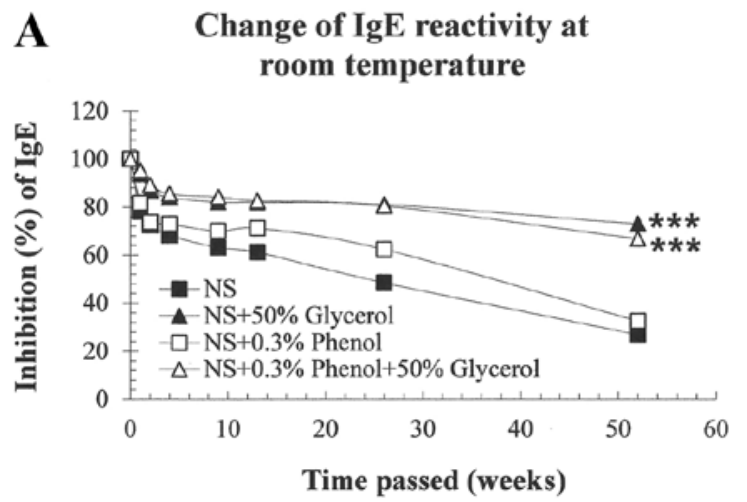

\section{B Change of IgE reactivity at $4^{\circ} \mathrm{C}$}

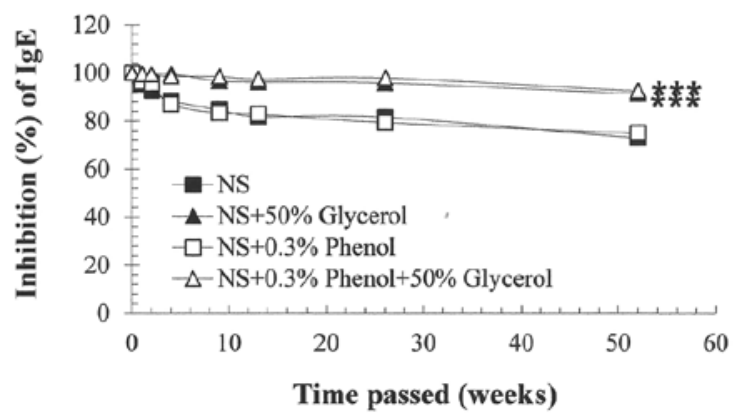

Figure 3. Change of extract potency over one year of storage under various conditions. IgE reactivity of the extracts was compared with the initial state by inhibition ELISA. Cockroach extracts were kept at (A) room temperature and (B) refrigerated. ${ }^{* * *} \mathrm{P}<0.001$. NS, normal saline. 

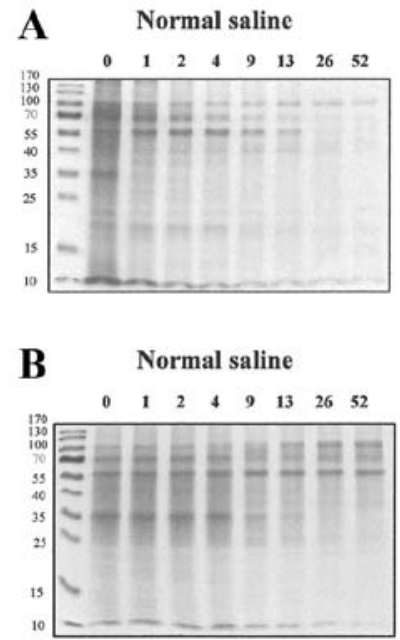

C Normal saline

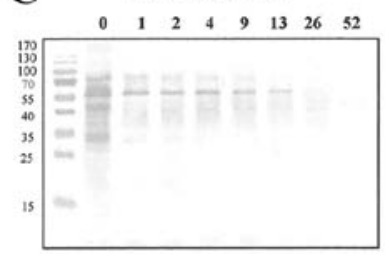

D

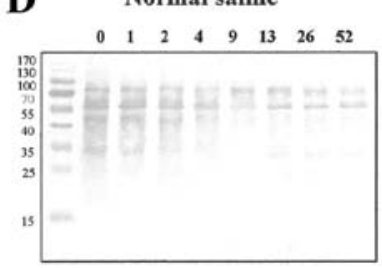

NS $+50 \%$ Glycerol

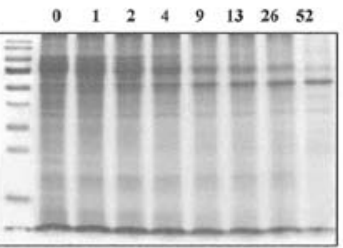

NS $+\mathbf{5 0} \%$ Glycerol

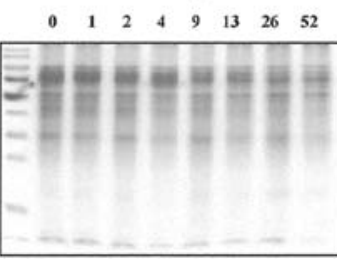

NS $+\mathbf{5 0} \%$ Glycerol

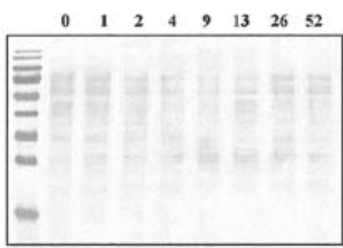

NS $+\mathbf{5 0} \%$ Glycerol

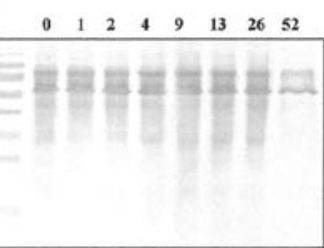

E
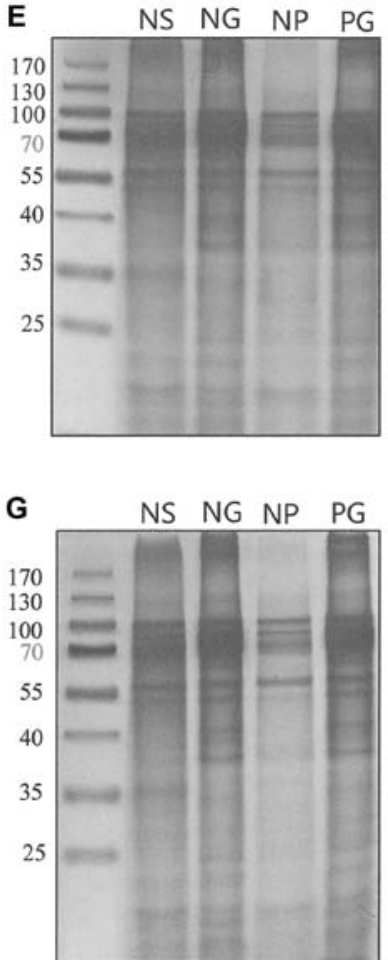

NS + $0.3 \%$ Phenol

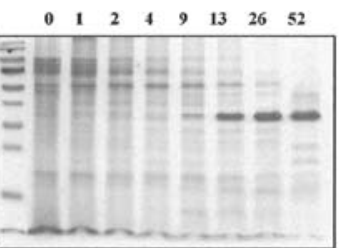

NS + 0.3\% Phenol

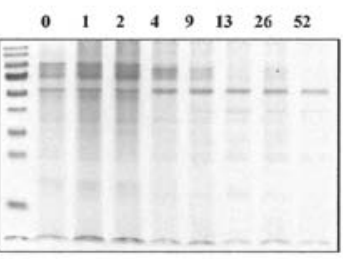

NS + $0.3 \%$ Phenol + $50 \%$ Glycerol

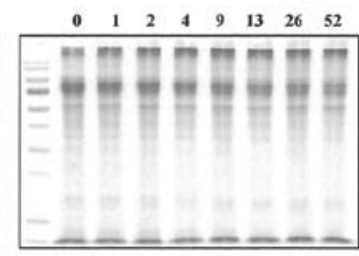

NS + $0.3 \%$ Phenol NS $+0.3 \%$ Phenol $+50 \%$ Glycerol
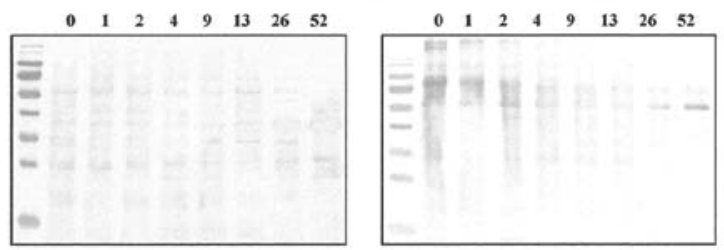

NS $+0.3 \%$ Phenol $\quad$ NS $+0.3 \%$ Phenol $+50 \%$ Glycerol
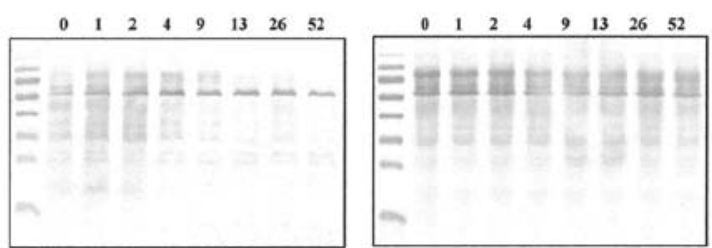
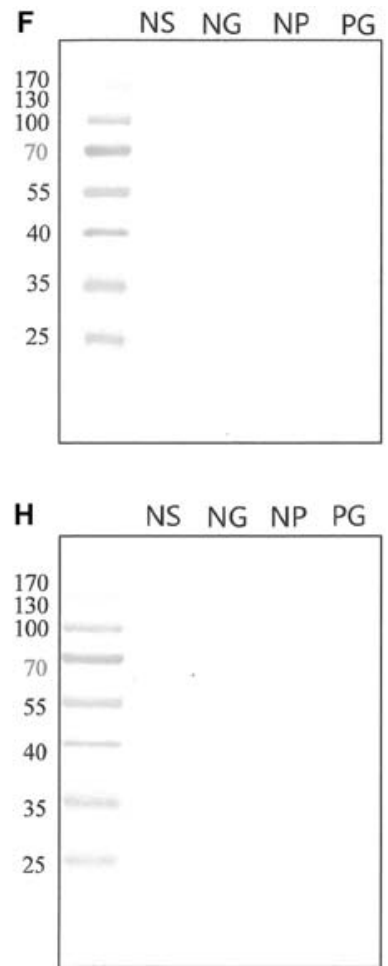

Figure 4. Change of protein profile and IgE reactive components over one year of storage in various conditions. SDS-PAGE of the extracts stored at (A) room temperature and (B) refrigerated. IgE immunoblotting of the extracts maintained at (C) room temperature and (D) refrigerated. The extracts stored at room temperature and refrigerated at week 0 were also examined by (E and G) SDS-PAGE and (F and H) IgE immunoblotting using a pooled serum sample from the healthy subjects as negative controls. NS, normal saline; NG, 50\% glycerol in normal saline; NP, $0.3 \%$ phenol in normal saline; PG, $0.3 \%$ phenol and $50 \%$ glycerol in normal saline. 
components in the extracts and produces high molecular weight bands in SDS-PAGE. However, these components were not allergenic, as examined by $\operatorname{IgE}$ immunoblotting. Furthermore, phenol, which is a reagent commonly used to eliminate protein in a laboratory, also influenced the banding pattern by SDS-PAGE. Some protein degradation by phenol may have caused the different protein profile and IgE reaction. The phenol effect is thought to be minimized by the addition of glycerol. In this study, Bla g 1 and Bla g 2 content in the cockroach extract were examined because two-site ELSIA kits for these allergens are commercially available. However, a growing body of evidence suggests that Bla $\mathrm{g} 1$ may not be a major allergen. None of the cockroach allergens identified to date appear to be immunodominant $(6,9,10)$. In recent studies, Bla g 5, Bla g 11 and Bla g 2 were determined as relatively important $(6,11)$. A more convenient and cheap assay for the quantification of Bla g 5 and Bla g 11 is required. Moreover, partially degraded allergens could retain IgE epitopes, although allergens could not be quantified with the two-site ELISA that recognizes two different epitopes. Liquid chromatography and multiple reaction monitoring mass spectrometry (LC-MRM MS) may offer rapid and accurate quantification of allergens. However, current LC-MRM MS technology is unlikely to detect and reflect the change in conformational epitopes in the extracts (12).

Extract potency is thought to better reflect extract reactivity stability for allergic reactions that are elicited by allergen-specific IgE antibodies. In general, potency was well preserved when $50 \%$ glycerol NS was added and refrigerated, as protein and allergen content were preserved. Notably, slower deterioration of potency was observed compared to the protein and allergen contents, reflecting the fact that some of the partially degraded allergens retained $\operatorname{IgE}$ reactivity.

Abrupt deterioration of cockroach extract in the absence of $50 \%$ glycerol NS is in line with the analyses of commercial aqueous and glycerinated cockroach extracts (13). On the other hand, the commonly used bacteriostatic agent phenol seems to have little effect on the stability of cockroach extract, although it has been shown to be disadvantageous over time with some pollen extracts (14).

The present study has several limitations. First, the effect of human serum albumin (HSA), a common reagent used as a stabilizer for allergen dilution, was not included. HSA is commonly added to protect allergen adsorption to glass vials and protein digestion. However, HSA is known to be effective in diluted extracts. Second, temperature excursions during shipping and clinical use were not tested; therefore, the results could be applied to the concentrated extract but not to diluted extracts. Finally, the change of protease activity over time was not examined. Proteases can elicit inflammatory reactions; however, allergenicity is not determined by protease activity but instead by IgE reactivity.

Taken together, cockroach extract with high protease activity can be stored for at least one year by the addition of $50 \%$ glycerol NS and refrigeration. The present study could facilitate the further refinement of allergen extract standardization with high protease activity and the development of improved diagnostics and immunotherapeutics. There is still great need for a surrogate system to assess the quality of cockroach extract.

\section{Acknowledgements}

Not applicable.

\section{Funding}

The present study was supported by a fund (grant no. 2015-ER6602-00) from the Research of Korea Centers for Disease Control \& Prevention. This research was also supported by a grant from the Korea Healthcare Technology R\&D Project through the Korean Health Industry Development Institute, which is funded by the Republic of Korea's Ministry of Health and Welfare (grant no. HI14C1324).

\section{Availability of data and materials}

The datasets used and/or analysed during the current study are available from the corresponding author on reasonable request.

\section{Authors' contributions}

KYJ drafted the manuscript. JL and JEY performed SDS-PAGE and IgE immunoblotting experiments. KHP, JHL and JWP collected serum samples. JDK prepared and provided cockroach extracts. KYJ, KHP, JHL and JWP designed the study and experiments. All authors read and approved the final manuscript.

\section{Ethics approval and consent to participate}

Patient consent was obtained before the blood collection. The Institutional Review Board of the Yonsei University Health System approved this study (no. 4-2013-0397).

\section{Patient consent for publication}

Not applicable.

\section{Competing interests}

KYJ, KHP, JHL, JDK and JWP have stocks in Prolagen. KYJ receives a consultancy fee from Prolagen.

\section{Authors' information}

$\mathrm{KYJ}$ is a technical advisor, JDK is a CEO, and JWP is a CTO of Prolagen.

\section{References}

1. Do DC, Zheo Y and Gao P: Cockroach allergen exposure and risk of asthma. Allergy 71: 463-474, 2016.

2. Nelson HS, Iklé D and Buchmeier A: Studies of allergen extract stability: The effects of dilution and mixing. J Allergy Clin Immunol 98: 382-388, 1996.

3. Sudha VT, Srivastava D, Arora N, Gaur SN and Singh BP: Stability of protease-rich Periplaneta americana allergen extract during storage: Formulating preservatives to enhance shelf life. J Clin Immunol 27: 294-301, 2007.

4. Grier TJ, Hall DM, Duncan EA and Goyne TC: Mixing compatibilities of Aspergillus and American cockroach allergens with other high-protease fungal and insect extracts. Ann Allergy Asthma Immunol 114: 233-239, 2015. 
5. Grier TJ, Hall DM, Duncan EA and Gada SM: Allergen stabilities and compatibilities in immunotherapy mixtures that contain cat, dog, dust mite, and cockroach extracts. Ann Allergy Asthma Immunol 115: 496-502, 2015.

6. Slater JE, James R, Pongracic JA, Liu AH, Sarpong S Sampson HA, Satinover SM, Woodfolk JA, Mitchell HE, Gergen PJ and Eggleston PA: Biological potency of German cockroach allergen extracts determined in an inner city population. Clin Exp Allergy 37: 1033-1039, 2007.

7. Jeong KY, Choi SY, Lee JH, Lee JS, Yong TS, Hong CS and Park JW: Preparation and characterization of an extract of German cockroach from a Korean source. Allergy Asthma Immunol Res 5: 102-105, 2013.

8. Plunkett G: Update: Stability of allergen extracts to establish expiration dating. Curr Opin Otolaryngol Head Neck Surg 24: 261-269, 2016

9. Satinover SM, Reefer AJ, Pomes A, Chapman MD Platts-Mills TA and Woodfolk JA: Specific IgE and IgG antibody-binding patterns to recombinant cockroach allergens. J Allergy Clin Immunol 115: 803-809, 2005.
10. Khurana T, Dobrovolskaia E, Shartouny JR and Slater JE: Multiplex assay for protein profiling and potency measurement of German cockroach allergen extracts. PLoS One 10: e0140225, 2015.

11. Jeong KY, Kim CR, Park J, Han IS, Park JW and Yong TS: Identification of novel allergenic components from German cockroach fecal extract by a proteomic approach. Int Arch Allergy Immunol 161: 315-324, 2013.

12. Mindaye ST, Spiric J, David NA, Rabin RL and Slater JE: Accurate quantification of 5 German cockroach (GCr) allergens in complex extracts using multiple reaction monitoring mass spectrometry (MRM MS). Clin Exp Allergy 47: 1661-1670, 2017.

13. Patterson ML and Slater JE: Characterization and comparison of commercially available German and American cockroach allergen extracts. Clin Exp Allergy 32: 721-727, 2002.

14. Niemeijer NR, Kauffman HF, van Hove W, Dubois AE and de Moncy JG: Effect of dilution, temperature, and preservatives on the long-term stability of standardized inhalant allergen extracts. Ann Allergy Asthma Immunol 76: 535-540, 1996. 